Pesq. Vet. Bras. 35(4):385-389, abril 2015 DOI: $10.1590 / \mathrm{S} 0100-736 \mathrm{X} 2015000400012$

\title{
Atividade da colinesterase plasmática como biomarcador de impacto ambiental em tartarugas verdes (Chelonia mydas) no litoral do Arquipélago de Fernando de Noronha, Pernambuco ${ }^{1}$
}

\author{
Leandro A. Fonseca ${ }^{2 *}$, Valéria Fagundes ${ }^{3}$, Fabrícia M. Girardi ${ }^{4}$, Natasha L. Maia ${ }^{4}$, \\ Filippe G. Pimentel ${ }^{4}$, Fábio R. Braga ${ }^{5}$, Emy Hiura ${ }^{5}$ e Marcelo R.D. Santos ${ }^{5}$
}

\begin{abstract}
Fonseca L.A., Fagundes V., Girardi F.M., Maia N.L., Pimentel F.G., Braga F.R., Hiura E. \& Santos M.R.D. 2015. [Plasma cholinesterase activity as environmental impact biomarker for green turtles (Chelonia mydas) on the coast of Fernando de Noronha, Pernambuco, Brazil.] Atividade da colinesterase plasmática como biomarcador de impacto ambiental em tartarugas verdes (Chelonia mydas) no litoral do Arquipélago de Fernando de Noronha, Pernambuco. Pesquisa Veterinária Brasileira 35(4):385-389. Departamento de Veterinária, Universidade Federal de Viçosa, Avenida P.H. Rolfs s/n, Campus Universitário, Viçosa, MG 36570-000, Brazil. E-mail: leandroabreu@ufv.br

Biomarkers can be used in a predictive manner, permitting actions control to be taken before they occur irreversible damage with severe environmental ecological consequences, however, sentinel species are needed to evaluate these markers. Sea turtles affected by fibropapillomas are considered sentinel species, signaling the marine environmental imbalance in their areas of occurrence. With the objective to use the plasma cholinesterase activity in green turtles (Chelonia mydas) as a biomarker, the measurement of enzyme activity was evaluated in healthy animals and in location of low pollution impact (Fernando de Noronha, Pernambuco, Brazil) to serve as a reference for comparison with animals trapped in places with higher pollution impact. Were analyzed heparinized plasma samples from 35 captured animals. All samples analyzed showed enzymatic activity of plasma cholinesterase. The values obtained ranged between 162$379 \mathrm{IU} / \mathrm{L}$, with mean and standard deviation of $216.4 \pm 51.4 \mathrm{IU} / \mathrm{L}$. In studies of repeatability and reproducibility were obtained variance coefficients of less than $5 \%$ in all analyzes, so the analytical methodology proved successful. The longevity of marine turtles of the species C. mydas, feeding behavior, along with the fact possess detectable enzyme activity may indicate this species as a bioindicator of exposure to pollutants that influence plasma cholinesterase activity.
\end{abstract}

INDEX TERMS: Biomarkers, green turtle, Chelonia mydas, cholinesterase.

RESUMO.- Biomarcadores podem ser usados de forma preditiva, permitindo que sejam tomadas ações de controle antes que ocorram danos ambientais irreversíveis com conse-

\footnotetext{
${ }^{1}$ Recebido em 4 de outubro de 2014.

Aceito para publicação em 28 de abril de 2015.

${ }^{2}$ Docente do Departamento de Veterinária, Universidade Federal de Viçosa (UFV), Avenida P.H. Rolfs s/n, Campus Universitário, Viçosa, MG 36570-000, Brasil. *Autor para correspondência: leandroabreu@ufv.br

${ }^{3}$ Docente do Departamento de Biologia Animal, Universidade Federal do Espírito Santo, Av. Marechal Campos, 1468, Maruípe, Vitória, ES 29043900, Brasil.

${ }^{4}$ Departamento de Veterinária, (UFV), Avenida P.H. Rolfs s/n, Campus Universitário, Viçosa, MG 36570-000, Brasil.

${ }^{5}$ Docente do Curso de Medicina Veterinária, Universidade Vila Velha (UVV), Av. Comissário José Dantas de Melo 21, Boa Vista, Vila Velha, ES 29102-920, Brasil.
}

quências ecológicas severas, no entanto, espécies sentinelas são necessárias para avaliação desses marcadores. As tartarugas marinhas são consideradas espécies sentinelas quando acometidas por fibropapilomas, sendo sinalizadora do desequilíbrio ambiental marinho nas suas áreas de ocorrência. Com o objetivo de propor a determinação da atividade da colinesterase plasmática em tartarugas verdes (Chelonia mydas) como biomarcador, procedeu-se a determinação da atividade enzimática em animais saudáveis e em localidade de baixo impacto antrópico (Arquipélago de Fernando de Noronha, Pernambuco, Brasil) para servir como referência para comparação com animais capturados em locais de maior impacto antrópico. Ao todo foram analisadas amostras de plasma heparinizado de 35 animais capturados. Todas as amostras analisadas apresentaram 
alguma atividade enzimática de colinesterase plasmática. Os valores obtidos de colinesterase variaram de 162 a 379 UI/L, com média e desvio padrão de 216,4 \pm 51,4 UI/L. Nos estudos de repetibilidade e reprodutibilidade obtiveram-se coeficientes de variação menor que 5\% em todas as análises, portanto a metodologia analítica utilizada se mostrou confiável. A longevidade das tartarugas marinhas da espécie C. mydas, o comportamento alimentar, juntamente com o fato de possuirem atividade enzimática detectável podem indicar essa espécie como bioindicadora de exposição a poluentes que influenciam na atividade da colinesterase plasmática.

TERMOS DE INDEXAÇÃO: Biomarcadores, tartaruga verde, Chelonia mydas, colinesterase.

\section{INTRODUÇÃO}

Atualmente, o crescimento industrial e urbano tem gerado impacto importante em alguns ecossistemas. Atividades industriais são responsáveis pela contaminação significativa de água, eliminando potenciais compostos sintéticos que atingem os rios e oceanos contribuindo assim, para o acúmulo de uma infinidade de produtos químicos em diversas concentrações (Freire et al. 2008). Por esse motivo, animais de ambientes marinhos costeiros tem sido alvo de severa ação antropogênica (Zwarg et al. 2014), com causas e efeitos ainda obscuros, dificultando a identificação de espécies "sentinelas" indicadoras de saúde ambiental (Aguirre \& Lutz 2004).

Alterações bioquímicas, celulares ou moleculares nas células, fluidos corpóreos, tecidos ou órgãos de um organismo podem ser utilizados como biomarcadores em respostas à exposição a alterações ambientais, comportamentais, psicológicas ou fisiológicas (Lam \& Gray 2003). Uma das características mais importantes dos biomarcadores é que sua avaliação antecipa mudanças nos altos níveis de organização biológica, isto é, população, comunidade ou ecossistema (Monserrat et al. 2007).

Desta forma, os biomarcadores podem ser usados de forma preditiva, permitindo que sejam tomadas ações de controle antes que ocorram danos ambientais irreversíveis com consequências ecológicas severas (Cajaraville et al. 2000). Os biomarcadores podem ser utilizados em estudos de campo que objetivam caracterizar áreas impactadas, onde uma complexa mistura de poluentes está normalmente presente (Monserrat et al. 2007).

As tartarugas marinhas são consideradas espécies sentinelas pois são acometidas pela fibropapilomatose, doença característica de ambientes poluídos e que é tida como um sinalizador do desequilíbrio ambiental marinho nas suas áreas de ocorrência (Aguirre \& Lutz 2004). A inibição da atividade da colinesterase tem sido usada como bioindicador para detectar e quantificar os efeitos biológicos de pesticidas organofosforados e carbamatos no ambiente aquático. Outros estudos afirmam que a colinesterase pode ainda, ser inibida por metais pesados e surfactantes (Coito et al. 2007). Este trabalho tem como objetivo determinar a atividade da colinesterase plasmática em tartarugas verdes (Chelonia mydas) no litoral do Arquipélago de Fernando de Noronha, Pernambuco, Brasil, para servir como referência para comparação com animais capturados em locais de maior impacto antrópico.

\section{MATERIAL E MÉTODOS}

O estudo foi realizado na Baia do Sueste, localizada no Arquipélago de Fernando de Noronha, estado de Pernambuco (3 ${ }^{\circ} 51^{\prime} 26^{\prime \prime} \mathrm{S}$ e $32^{\circ} 25^{\prime} 33^{\prime \prime} 0$ ). A região possui duas Unidades de Conservação Federais, uma Área de Proteção Ambiental (APA) e o Parque Nacional Marinho e é caracterizada como uma importante área de alimentação e desova de Chelonia mydas (Babptistotte 2007). Pelo fato dos animais estarem em mar aberto, a captura manual teve perseguição contínua, prolongada, sendo necessário um tempo de mergulho e perseguição superiora 60 minutos, até a completa captura do indivíduo e seu transporte para a praia. As licenças para coleta, transporte e manipulação do material biológico foram concedidas pelo Sistema de Autorização e Informação em Biodiversidade (SISBIO)/ICMBio - IBAMA, sob registro número 26080. Foram registrados de cada animal, as medidas de comprimento curvilíneo da carapaça (CCC), largura curvilínea da carapaça (LCC) e peso. Os que foram encontrados sem identificação tiveram a colocação de marcas de monelinconel (National Band and Tag Co., USA) nas nadadeiras dianteiras, conforme metodologia padrão adotada pelo Projeto ICM-Bio/IBAMA (Marcovaldi \& Marcovaldi 1999). 0 sexo dos animais não foi determinado devido à ausência de dimorfismo sexual externo nessa fase do ciclo de vida.

A coleta de sangue periférico foi realizada por venopunção do seio venoso cervical ou veia jugular, precedido de limpeza e antissepsia adequadas com álcool iodado. 0 sangue foi transferido imediatamente para um tubo contendo $5 \mathrm{ml}$ de heparina (Vacutainer ${ }^{\circledR}$ ), mantido entre 2 e $8^{\circ} \mathrm{C}$ até o início do processamento, não excedendo 48 horas até o início das dosagens.

Para medir a atividade da enzima colinesterase utilizou-se o método de Ellman et al. (1961), modificado por Sturm et al. (1999), que permite quantificar a atividade da enzima em amostras de plasma. A determinação da atividade plasmática da colinesterase foi realizada por método enzimático cinético. A reação ocorre devido a ação catalítica da colinesterase na hidrolização da butiriltiocolina em tiocolina e butirato. Dessa forma, a tiocolina reduz o hexacianoferrato III (que apresenta cor amarela), em hexacianoferrato II (incolor). 0 decréscimo da absorbância é medido a $405 \mathrm{~nm}$ e representa a intensidade da atividade enzimática. Para essa dosagem o teste apresenta limite mínimo de detecção de 50 U/L e a reação é linear até 25000 U/L.

Para análise estatística da dosagem de colinesterase foi realizado previamente um estudo de repetibilidade e reprodutibilidade das amostras coletadas. Para a realização do teste de repetibilidade do método, procedeu-se a medição da atividade da colinesterase plasmática nas amostras de sangue de C. Mydas, efetuando 10 réplicas sucessivas nas mesmas condições de medição. Para a realização do teste de reprodutibilidade do método de dosagem da atividade enzimática, procedeu-se a medição nas amostras mantidas a $-20^{\circ} \mathrm{C}$, variando a condição tempo (dia de medição/número de dias a $-20^{\circ} \mathrm{C}$ ).

Para avaliar os testes de repetibilidade e reprodutibilidade, determinou-se o erro padrão e o intervalo de confiança associada ao método. Para o cálculo do intervalo de confiança, utilizou-se um grau de confiança de $95 \%$. Além disso, o coeficiente de variação menor que $5 \%$ foi utilizado como um critério para avaliar a repetibilidade e a reprodutibilidade do método.

\section{RESULTADOS}

Todos os animais capturados eram juvenis conforme critérios propostos por Hirth (1997), ou seja, comprimento cur- 
vilíneo de carapaça (CCC) menor que $73,5 \mathrm{~cm}$. Os animais apresentaram CCC variando de 28,4 a $56,3 \mathrm{~cm}$, com média e desvio padrão de 51,8 $\pm 11,3 \mathrm{~cm}$ (Fig.1).

Ao todo foram analisadas amostras de plasma heparinizado de 35 animais capturados. Todas as amostras analisadas apresentaram alguma atividade enzimática de colinesterase plasmática (Fig.2). Os valores obtidos de colinesterase variaram de 162 a 379 UI/L, com média e desvio padrão de 216,4 $\pm 51,4 \mathrm{UI} / \mathrm{L}$.

Para o estudo de repetibilidade e reprodutibilidade, foram utilizadas amostras de três dos indivíduos capturados, escolhidos aleatoriamente. Os resultados encontrados no estudo de repetibilidade estão representados no Quadro 1 , respectivamente, para as dez réplicas efetuadas de cada indivíduo. Os resultados encontrados no estudo de reprodutibilidade estão representados no Quadro 2, respectivamente, para os quatro dias de avaliação.

Nos estudos de repetibilidade e reprodutibilidade obtiveram-se coeficientes de variação menor que $5 \%$ em todas as análises, portanto a metodologia analítica utilizada se mostrou confiável. A repetibilidade expressa a fidelidade obtida nas mesmas condições operacionais, aplicadas em um curto intervalo de tempo pós coleta (inferior à 12 horas pós coleta, sob refrigeração). A reprodutibilidade designa a fidelidade da análise num intervalo de tempo, geralmente obtida em análises diárias. A principal função da reproduti-

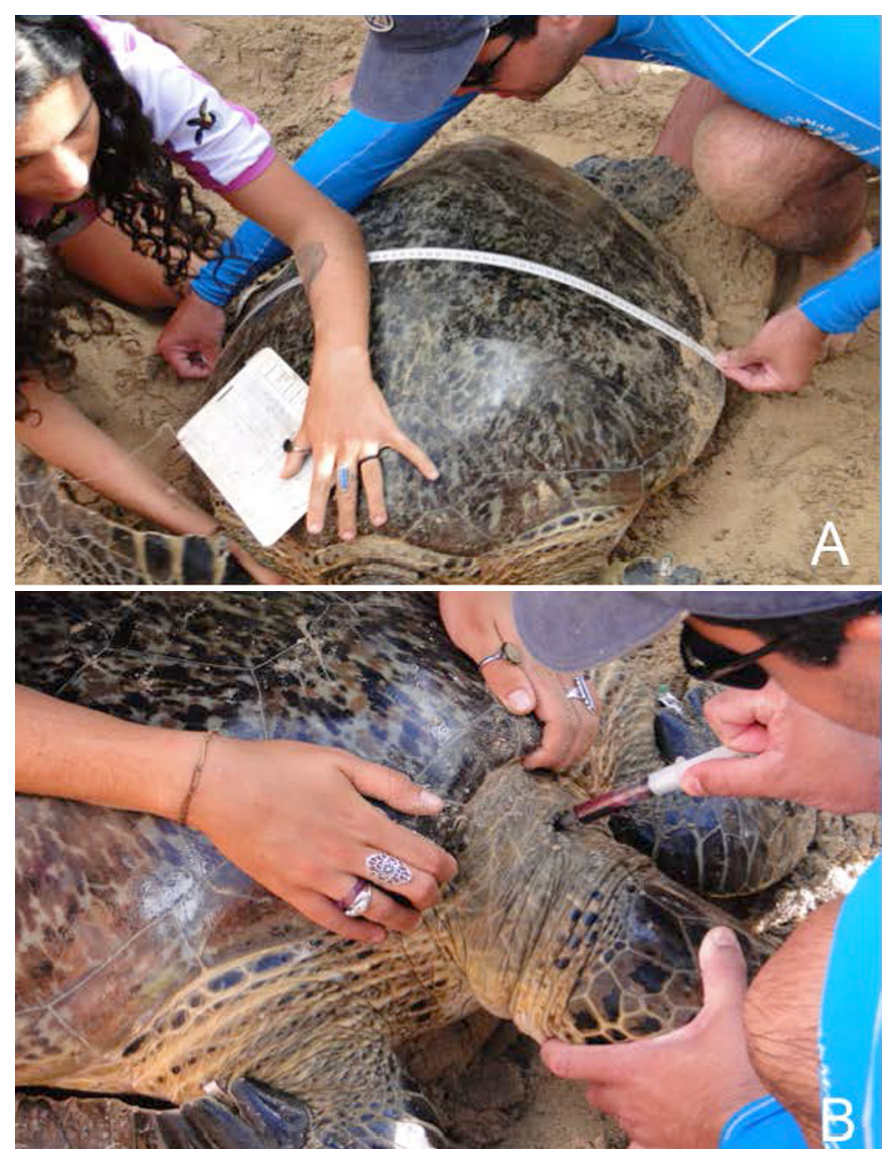

Fig.1. Manejo dos animais após a captura na Baia do Sueste, Fernando de Noronha. (A) Identificação e coleta de dados do animal capturado, (B) Coleta de sangue em seio venoso cervical para análise.

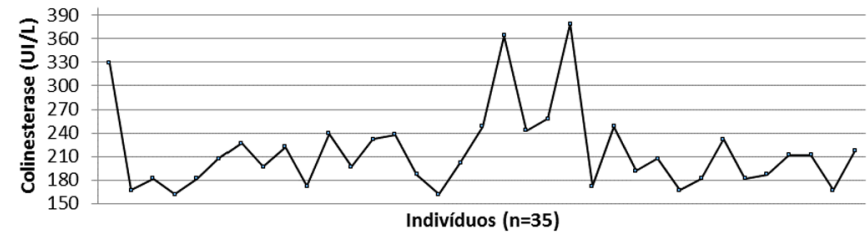

Fig.2. Demonstração da atividade da colinesterase plasmática em tartaruga marinha da espécie Chelonia mydas $(\mathrm{n}=35)$ capturadas no Arquipélago de Fernando de Noronha, Pernambuco, Brasil.

Quadro 1. Resultado do estudo de repetibilidade da atividade da colinesterase plasmática em tartaruga marinha (Chelonia mydas)

\begin{tabular}{lccc}
\hline \multicolumn{4}{c}{ Estudo de reprodutibilidade } \\
\hline Ensaio & \multicolumn{2}{c}{ Dosagem da atividade da colinesterase (UI/L) } \\
\cline { 2 - 4 } & Animal 1 & Animal 2 & Animal 3 \\
\hline 1 & 329 & 167 & 182 \\
2 & 328 & 165 & 182 \\
3 & 328 & 167 & 181 \\
4 & 329 & 167 & 182 \\
5 & 329 & 167 & 182 \\
6 & 329 & 166 & 181 \\
7 & 329 & 168 & 180 \\
8 & 328 & 167 & 181 \\
9 & 328 & 167 & 182 \\
10 & 329 & 166 & 182 \\
Média & 328,6 & 166,7 & 181,5 \\
Desvio Padrão & 0,5 & 0,8 & 0,7 \\
Erro Padrão & 0,15 & 0,25 & 0,22 \\
Intervalo & $328,24-328,95$ & $166,12-167,27$ & $180,99-182,00$ \\
de Confiança & & & \\
Coeficiente & $0,15 \%$ & $0,47 \%$ & $0,37 \%$ \\
de Variação & & &
\end{tabular}

Quadro 2. Resultado do estudo de reprodutibilidade da atividade da colinesterase plasmática em tartaruga marinha (Chelonia mydas)

\begin{tabular}{lccc}
\hline \multicolumn{4}{c}{ Estudo de reprodutibilidade } \\
\hline Ensaio & \multicolumn{2}{c}{ Dosagem da atividade da colinesterase (UI/L) } \\
\cline { 2 - 4 } & Animal 1 & Animal 2 & Animal 3 \\
\hline Dia 1 & 329 & 167 & 182 \\
Dia 2 & 325 & 167 & 181 \\
Dia 3 & 325 & 164 & 180 \\
Dia 4 & 321 & 162 & 178 \\
Média & 325,0 & 165,0 & 180,3 \\
Desvio Padrão & 2,8 & 2,1 & 1,5 \\
Erro Padrão & 1,4 & 1,05 & 0,75 \\
Intervalo & $320,54-329,45$ & $161,65-168,34$ & $177,91-182,68$ \\
de Confiança & & & \\
Coeficiente & $0,87 \%$ & $1,29 \%$ & $0,82 \%$ \\
de Variação & & &
\end{tabular}

bilidade é confirmar se determinada metodologia pode ou não ser executada em dias diferentes pós coleta, gerando resultados confiáveis dentro dos critérios estabelecidos. Assim, enquanto o termo repetibilidade se refere à precisão do método, a reprodutibilidade se refere à precisão na estabilidade da atividade enzimática.

\section{DISCUSSÃO}

Das tartarugas marinhas avaliadas nesse trabalho, observou-se que todas apresentaram alguma atividade da coli- 
nesterase plasmática. Entretanto, existem poucos estudos sobre contaminantes ambientais em répteis, se restringindo à efeitos de metais pesados em quelônios (Aguirre 1994). A maioria dos trabalhos relatando a atividade da colinesterase tratam do assunto em espécies de peixes (Jesus et al. 2013).

Segundo Baatrup (1991) a presença de chumbo no organismo de peixes causa aumento dos níveis de histamina, serotonina, lipídeos, colesterol e proteínas séricas; mas causa a diminuição do GABA e da colinesterase plasmática. 0 chumbo pode ser incorporado através da inalação de vapores ou ingestão de sais de chumbo, principal via de exposição dos animais. Após absorvido, este metal é distribuído pelos tecidos orgânicos e se deposita, principalmente, nos enterócitos e nos ossos. Por competir diretamente com o cálcio, deficiências nutricionais podem aumentar a absorção deste contaminante. Em nível celular, essa competição pode levar a alterações de processos celulares dependentes de cácio (como canais de cálcio voltagem-dependentes), a inativação de sistemas enzimáticos e a interferência na transmissão nervosa por colinesterses e dopamina, por exemplo (Hueza et al. 2008).

Ainda, metais pesados e outros poluentes químicos, prejudicam o sistema imune e provocam estresse em animais e no homem. $\mathrm{O}$ efeito mediado por poluentes químicos causa mudanças morfofísiológicas como a proliferação celular (tumor de pele, por exemplo, que tem sido documentado frequentemente algumas espécies de tartarugas marinhas) e maior predisposição a agentes infecciosos (Barbieri 2009).

A inibição da colinesterase plasmática tem sido utilizada como parâmetro indicativo de exposição a compostos organofosforados e carbamatos, porém a sua relação com metais pesados e organoclorados tem sido pouco estudada (Jesus et al. 2013). Detergentes e hidrocarbonetos são substâncias que podem conter inibidores da colinesterase plasmática. No entanto, pouco se sabe sobre a relação entre as propriedades cinéticas da colinesterase de organismos aquáticos e o efeito inibitório de poluentes com ação anticolinesterásica (Monserrat \& Bianchini, 2001).

Existem duas formas de se utilizar a atividade plasmática da colinesterase como bioindicadora. A primeira, é quantificar os indivíduos que apresentarem diminuição da atividade enzimática equivalente a duas vezes o desvio padrão da média obtida de todos os animais avaliados. A segunda forma, é identificar os animais que apresentarem diminuição da atividade equivalente a $20 \%$ da média obtida de todos os animais (Meyer et al. 2013).

Vários estudos avaliando a atividade da colinesterase em outras espécies foram realizados, porém, nenhum utilizando tartarugas marinhas. Lajmanovich et al. (2004) observaram diminuição significativa da atividade enzimática em sapos (Bufo paracnemis) capturados em uma área rurais de agricultura quando comparados com outros capturados em uma área controle. Sanchez-Hernandez (2004) encontrou diminuição significativa da enzima em lagartos (Gallotia galloti palmae) que ocupavam um local de agricultura nas Ilhas Canárias. Meyer et al. (2013) realizaram o primeiro estudo em tartarugas de água doce (Emys marmorata) e encontrou diminuição da atividade enzimática em animais influenciados por contaminação por pesticidas.
Segundo Gardner (2006), estudos realizados em áreas de alimentação ocupadas por juvenis de C. mydas em diferentes regiões do globo sugerem que determinadas populações são fiéis aos seus sítios de forrageamento até atingirem a fase adulta, quando migram para outras áreas de alimentação onde a oferta de recursos alimentares é mais adequada ao novo hábito alimentar de herbivoria. Esse hábito, juntamente ao fato de possuirem atividade detectável da colinesterase plasmática, pode sugerir essa espécie como importante bioindicadora de exposição à metais pesados que influenciam na atividade da colinesterase, principalmente em ambientes de impacto industrial.

Ainda segundo Aguirre (1994), os efeitos dos metais pesados no organismo de tartarugas-marinhas são desconhecidos devido a escassez de pesquisas desses elementos em répteis aquáticos. Torna-se, então, difícil de interpretar a significância dos valores encontrados em relação aos níveis de normalidade nesses animais.

A longevidade das tartarugas marinhas da espécie $C$. mydas, o comportamento alimentar herbívoro, juntamente com o fato de possuirem atividade detectável da colinesterase plasmática, podem indicar essa espécie como bioindicadora de exposição a poluentes que influenciam nessa atividade enzimática, principalmente em ambientes sob influência de atividades industriais. Para isso é necessário avaliar a atividade enzimática sob diferentes condições e exposições, tanto in situ por comparação entre locais quanto, ex situ, através de bioensaios. A possibilidade de se utilizar amostras sanguíneas possibilita o desenvolvimento de estudos pouco invasivos e que permitam essa análise minimizando os óbices éticos do uso de animais em pesquisa.

Por fim, os resultados obtidos no presente estudo são pioneiros e importantes, uma vez que poderão ser utilizados como referência para trabalhos futuros de trabalhos de biomonitoramento dessa espécie.

Agradecimentos.- À Fundação de Amparo à Pesquisa do Espírito Santo (FAPES), à Fundação de Amparo à Pesquisa do Estado de Minas Gerais (FAPEMIG) e ao Conselho Nacional de Desenvolvimento Científico e Tecnológico $(\mathrm{CNPq})$ pelo apoio.

\section{REFERÊNCIAS}

Aguirre A.A. 1994. Organic contaminants and trace metals in the tissues of green turtles (Chelonia mydas) afflicted with fibropapillomas in the Hawaiian Islands. Mar. Pol. Bul. 28:109-114

Aguirre A.A. \& Lutz P.L. 2004. Marine turtles as sentinels of ecosystem health: is fibropapillomatosis an indicator? EcoHealth 3:275-283.

Baatrup E. 1991. Structural and functional effects ofheavy metais on the nervous system, including sense organs, of fish. Comp. Biochem. Physiol. 100:253-257.

Baptistotte C. 2007. Caracterização espacial e temporal da fibropapilomatose em tartarugas marinhas da costa brasileira. Tese de Doutorado em Ecologia Aplicada, Escola Superior de Agricultura Luiz de Queiroz, Universidade de São Paulo, Piracicaba, SP. 60p.

Barbieri E. 2009. Concentration of heavy metals in tissues of green turtles (Chelonia mydas) sampled in the Cananéia estuary, Brazil. Braz. J. Oceanog. 57:243-248.

Cajaraville M.P., Bebianno M.J. \& Blasco J. 2000. The use of biomarkers to assess the impact of pollution in coastal environments of the Iberian Peninsula: a practical approach. The Science of the Total Environment 
247:295-311.

Coito R., Torres P. \& Costa M.C. 2007. Actividade de acetilcolinesterase em esponjas marinhas da costa portuguesa. Revta Lusóf. Ciênc. Tecnol. Saúde 4:202-214.

Ellman G.L., Courtney K.D., Andres Jr V. \& Featherstone R.M. 1961. A new and rapid colorimetric determination of acetylcholinesterase activity. Biochem. Pharmacol. 7:88-90.

Freire M.M., Santos V.G., Ginuino I.S.F. \& Arias A.R.L. 2008. Biomarcadores na avaliação de saúde ambiental dos ecossistemas aquaticos. Oecol. Bras. 12:347-354.

Hirth H.F. 1997. Synopsis of the biological data on the green turtle Chelonia mydas (Linnaeus 1758). Fish and Wildlife Service, U.S. Department of the Interior, Washington, D.C.

Hueza I.M., Sant'Ana M.G. \& Palermo-Neto J. 2008. Toxicologia do chumbo, mercúrio, arsênio e outros metais, p.641-662. In: Spinosa H.S., Górniak S.L. \& Palermo-Neto J. (Eds), Toxicologia Aplicada à Medicina Veterinária. Manole, São Paulo.

Jesus T.B., Colombi J.S., Ribeiro C.A.O., Assis H.C.S. \& Carvalho C.E.V. 2013. Cholinestarase activity in methylmercury and mercury chloride exposure fish. Ecotoxicol. Environ. Contam. 8:147-148.

Lajmanovich R.C., Sanchez-Hernandez J.C., Stringhini G. \& Peltzer P.M. 2004. Levels of serum cholinesterase activity in the rococo toad (Bufo paracnemis) in agrosystems of Argentina. Bull. Environ. Contam. Toxicol. 72:586-591.

Lam P.K.S. \& Gray J.S. 2003. The use of biomarkers in environmental monitoring programmes. Marine Pollution Bull. 46:182-186.
Marcovaldi M.A. \& Marcovaldi G.G. 1999. Marine turtles of Brazil: the history and structure of Projeto Tamar-Ibama. Biol. Conserv. 91:35-41.

Meyer E., Sparling D. \& Blumenshine S. 2013. Regional inhibition of cholinesterase in free-ranging western pond turtles (Emys marmorata) occupying california mountain streams. Environ. Toxicol. Chem. 32:692-698.

Monserrat J.M. \& Bianchini A. 2001. Anticholinesterase effect of eserine (physostigmine) in fish and crustacean species. Braz. Arch. Biol. Technol. 44:63-68.

Monserrat J.M., Martinez P.E. \& Geracitano L.A. 2007. Pollution biomarkers in estuarine animals: critical review and new perspectives. Comp. Biochem. Physiol. C, Toxicol. Pharmacol. 146:221-34.

Gardner S.C. 2006. Heavy metals accumulation in four species of sea turtles from the Baja California peninsula, Mexico. BioMetals, London, 19:91-99.

Sanchez-Hernandez J.C., Carbonell R., Henriquez Perez A., Montealegre M. \& Gomez L. 2004. Inhibition of plasma butyryl cholinesterase activity in the lizard Gallotia galloti palmae by pesticides: a field study. Environ. Pollut. 132:479-488.

Sturm A., Silva de Assis H.C. da \& Hansen P.-D. 1999. Cholinesterases of marine teleost fish: enzymological characterization and potential use in the monitoring of neurotoxic contamination. Mar. Environ. Res. 47:389398.

Zwarg T., Rossi S., Sanches T.C., Cesar M.O., Werneck M.R. \& Matushima E.R. 2014. Hematological and histopathological evaluation of wildlife green turtles (Chelonia mydas) with and without fibropapilloma from the north coast of São Paulo State, Brazil. Pesq. Vet. Bras. 34:682-688. 